\section{Rare variant association studies}

Eric Lander and colleagues report an analysis offering considerations for the design of rare variant association studies (Proc. Natl. Acad. Sci. USA 111, E455-E464, 2014). They consider a binary trait, exemplifying such a disease phenotype, analyzed with burden tests and use a two-class model in which they assume that alleles are either null, abolishing gene function, or neutral, not accounting for intermediate effects. Their simulations estimate the sample sizes needed to detect rare variant associations across a range of study designs, demonstrating a strong dependence on the mutation rate, selection coefficient and effect sizes for null alleles in a gene. For rare variants in coding regions, their first strategy is to consider only disruptive variants in each gene. They suggest that missense alleles may also be included to increase power, but care should be taken to filter using optimally selected frequency thresholds and experimental or computational predictions. They also consider the benefits of studying isolated populations with recent bottlenecks. For noncoding regions, the authors note that a detailed understanding of functionally relevant regulatory sequences for each gene is needed. For current rare variant association studies, they recommend the use of exome, rather than whole-genome, sequencing to maximize power to detect association.

\section{Molecular barcodes improve RNA-seq}

Stephen Fodor, Ronald Davis and colleagues have developed a cDNA barcoding method to estimate the efficiency of library preparation for RNA sequencing (RNA-seq) and to allow for more accurate quantification of transcript levels (Proc. Natl. Acad. Sci. USA 111, 1891-1896, 2014). The method uses a two-step process to generate cDNA libraries in which each molecule of any given cDNA sequence is labeled with a unique combination of barcodes. The authors use their labeled libraries to obtain absolute quantification of mRNA species after sequence capture enrichment for targeted RNA-seq, thus improving the ability to detect low-copy number transcripts while adjusting for error introduced during the amplification steps. Finally, the authors demonstrate that standard procedures for creating cDNA libraries for use in RNAseq have extremely low efficiency. By spiking in a set of 960 barcoded synthetic RNAs, they were able to track the loss of input RNA through multiple steps of library creation. Overall, approximately 2-3 copies of a transcript remained in the completed library for every 1,000 copies in the input sample. These results demonstrate the need for improved methods of cDNA library preparation.

$B L$

\section{Protective role for beige fat}

In mice, beige adipose cells emerge from subcutaneous white adipose tissue in response to stimuli such as cold or $\beta$-adrenergic agonists, but their physiological role has not been fully elucidated. To explore this question, Bruce Spiegelman and colleagues generated mice in which the function of beige fat cells was selectively impaired by conditional deletion of a key transcriptional regulator, $\operatorname{Prdm} 16$, from adipocytes (Cell 156, 304-316, 2014). When fed normal chow, these mice showed

Written by Orli Bahcall, Brooke LaFlamme, Emily Niemitz \& Kyle Vogan no differences in food intake, activity or oxygen consumption. However, when challenged with a high-fat diet, they developed severe insulin resistance and obesity, accompanied by hepatic steatosis and a substantial increase in subcutaneous fat mass. Notably, the subcutaneous adipose tissue from these mice exhibited a gene expression profile resembling that of visceral fat, with reduced expression of thermogenic genes such as $U c p 1$ and elevated expression of proinflammatory genes. Together, these findings indicate that the Prdm16-dependent thermogenic program of beige adipose cells helps protect mice from adverse metabolic changes in response to a high-fat diet, and they provide further insights into the relationship between body fat distribution and disease risk.

$K V$

\section{Parent-of-origin effects}

The extent to which parent of origin (the inheritance of genetic variation from the mother or father) contributes to the heritability of complex traits is unknown. Now, Richard Mott and colleagues use data from a mouse heterogeneous stock population to show that the majority of 97 complex traits have measurable parent-of-origin effects (Cell 156, 332-342, 2014). Heterogeneous stock mice were bred from 8 inbred progenitor strains and maintained for over 50 generations. The authors used available phenotype and genotype data to measure parent-of-origin effects on trait heritability, finding such effects for 91 of 97 (93\%) traits. The authors analyzed 837 known quantitative trait loci (QTLs) for the 97 traits and found that QTLs with parentof-origin effects included imprinted loci and loci not known to be imprinted. They further investigated two QTLs not known to be imprinted by selecting a candidate gene within each QTL and performing reciprocal crosses of knockout alleles. These analyses confirmed that Man1a2 has parent-of-origin effects on body weight and that $\mathrm{H} 2-a b 1$ has parent-of-origin effects on a percentage of $\mathrm{CD} 4^{+} \mathrm{T}$ cells. The authors suggest that parent-of-origin effects may be generated by altering in trans the expression of imprinted genes. EN

\section{Poor sleep and tumor progression}

An abnormal pattern of repeated arousals during sleep, known as sleep fragmentation, is a key characteristic of sleep apnea and has been associated with increased risk of cancer development and mortality. Now, David Gozal and colleagues identify a gene, Toll-like receptor 4 (TIr4), that drives increased aggressiveness of tumors in mice with fragmented sleep (Cancer Res. doi: 10.1158/0008-5472.CAN-13-3014, 21 January 2014). The researchers split the mice into two groups: a control group allowed to sleep normally during the day (from 7 a.m. to 7 p.m.) and an experimental group woken every 2 min by sweeping a near-silent motorized brush through their cage to simulate sleep fragmentation. One week after starting the treatments, mice were injected with cultured tumor cells. After $28 \mathrm{~d}$, the mice with fragmented sleep had larger, more invasive tumors than their wellrested counterparts. The mice with sleep fragmentation also had higher counts of tumor-associated macrophages and higher levels of TLR4, suggesting a role for the immune system. Importantly, knockout of Tlr4 abolished these differences between control mice and ones with sleep fragmentation. These results suggest a potential mechanism by which disturbed sleep in humans affects tumor progression and point to a therapeutic target for cancer patients with sleep disturbance. 